\title{
Immunotherapy of breast cancer by single delivery with rAAV2-mediated interleukin-15 expression
}

\author{
YUNG-LUEN YU ${ }^{1,2 *}$, CHYOU-WEI WEI ${ }^{3 *}$, YI-LIN CHEN ${ }^{4 *}$, \\ MARK HUNG-CHIH CHEN $^{5}$ and GIOU-TENG YIANG ${ }^{6}$
}

\begin{abstract}
${ }^{1}$ Graduate Institute of Cancer Biology and Center for Molecular Medicine, China Medical University and Hospital; ${ }^{2}$ Department of Biotechnology, Asia University, Taichung; ${ }^{3}$ Institute of Biomedical Nutrition, College of Medicine and Nursing, Hungkuang University, Sha Lu, Taichung; ${ }^{4}$ Graduate Institute of Biotechnology, National Ilan University, Ilan;

${ }^{5}$ Department of Biotechnology, Hungkuang University, Sha Lu, Taichung; ${ }^{6}$ Department of Emergency Medicine, Tzu Chi University and Buddhist Tzu Chi General Hospital, Hualien, Taiwan, R.O.C.
\end{abstract}

Received September 24, 2009; Accepted November 9, 2009

DOI: 10.3892/ijo_00000508

\begin{abstract}
Recombinant adenovirus-associated vector serotype 2 (rAAV2) is one of the most promising gene transfer vectors due to its advantage of causing non-pathogenic infection, low immunogenicity, and long-term gene expression in human clinical trials. Human interleukin 15 (hIL15) has been implicated in modulation of antitumor activity of lymphokine-activated killer (LAK) cells, including T cells and NK cells. In this study, the rAAV2-hIL15 vector was produced and subjected for treatment with xenograft JC breast cancer model. Results showed that tumor onset was significantly delayed, the tumor growth was suppressed, and the lifespan of tumor-bearing mice were prolonged by rAAV2-hIL15. In addition, rAAV2-hIL15 was able to produce a substantial expression of IL15 protein that ultimately activated the cytotoxic activity of LAK cells. Furthermore, prominent apoptosis was observed in tumor lesions following injection of rAAV2-hIL15. Taken together, our results suggested that rAAV2-hIL15 appears as a new potential therapeutic tool for breast cancer immunotherapy.
\end{abstract}

Correspondence to: Dr Giou-Teng Yiang, Department of Emergency Medicine, Buddhist Tzu Chi General Hospital, Hualien, Taiwan, R.O.C.

E-mail: jtyiang@ms73.hinet.net

Dr Chyou-Wei Wei, Institute of Biomedical Nutrition, College of Medicine and Nursing, Hungkuang University, Sha Lu, Taichung, Taiwan, R.O.C.

E-mail:wcwnina@gate.sinica.edu.tw

*Contributed equally

Key words: rAAV2, hIL15, immunotherapy, breast cancer, LAK cells

\section{Introduction}

The adenovirus-associated vectors (AAV) are known to have the best safety profile among viral vectors without causing any known disease and exhibit low immunogenicity. In particular, recombinant AAV serotype 2 (rAAV2) is able to infect both dividing and non-dividing cells while maintaining long-term gene expression up to more than a year in a broad range of tissues, including liver, brain, retina, and muscle $(1,2)$. rAAV2 have been applied to cancer gene therapy and showed a great deal of promise as a viral vector, delivering therapeutic genes such as antiangiogenesis associated genes (e.g., angiostatin) (3) and immune regulatory genes (e.g., interferon) (4) or by vaccination (5). Among the diversified AAV serotypes, AAV2 is well characterized, and it has been employed in clinical trials of cystic fibrosis, hemophilia, and Canavan's disease (6). More recently, rAAV vector expressing glutamic acid decarboxylase (GAD) gene was successfully applied to the treatment of patients who had advanced Parkinson's disease with no adverse effects or immunogenic reactions $(7,8)$. These data further emphasized the safety and efficacy of rAAV for gene therapy in human.

Breast cancer is a complex and multifactorial disease, it can span decades during the period of development and clinical course is highly variable. Both the innate and acquired arms of the immune system are believed to play crucial roles in the antitumor response. Cytokines including interleukins (IL1, IL2, IL10, IL12, IL18, IL24), tumor necrosis factor- $\alpha$ (TNF- $\alpha$ ), and the interferon- $\gamma(\mathrm{IFN}-\gamma)$ all play a key role in controlling the immune response and with a potential role in immunotherapy of breast cancer $(9,10)$ and the cytokines IL2, IFN- $\gamma$, and TNF- $\alpha$ have also been approved to treat advanced melanoma, renal cell carcinoma (RCC), and sarcoma (10).

IL15 is a $14-15-\mathrm{kDa}$ immunostimulatory cytokine with structural similarity to IL2 that is secreted by mononuclear phage upon infection by viruses $(11,12)$. This cytokine induces cell proliferation and differentiation of $\mathrm{T}$ cells, B cells and NK cells, whose principal role is to kill pathogenic infected cells, activates innate immune defense system (11-14) and 
was found to be superior to IL2 in generating cytolytic effector splenocytes (12). In addition, both IL2 and IL15 are able to stimulate antitumor cytotoxic activities of cytotoxic $\mathrm{T}$ cells (CTL) and NK cells, and these two kinds of lymphocytes can further develop into lymphokine-activated killer (LAK) cells $(15,16)$. Immunotherapy with LAK cells has been widely used in cancer therapy, including breast, lung, ovary, and pancreatic cancers in phase II clinical trials (17). For its features in activation of the immune system, IL15 was considered a good candidate for cancer immunotherapy $(12,18)$.

The current situation with regards to the use of cytokines in immunotherapy is that many studies used cytokine proteins to treat cancer patients through multi-dose daily injections (up to three doses per day) because of their short lives (17) and also injection of cytokine genes into tumor cells was documented $(19,20)$. The methods described above are not feasible to use for clinical purpose due to high expense and complexity of the injection procedure. In order to deal with these hindrances, we are trying to produce rAAV2 carrying human IL15 (hIL15) gene for cancer immunotherapy. In our previous study, we successfully inhibited HeLa cell tumor growth by using rAAV2-hIL15 (21). In the present study, we further produced rAAV2-hIL15 to treat and inhibit xenografted JC breast cancer cells in a syngenic BALB/c mice model. Our results may provide a new potential safe and effective therapeutic tool for breast cancer immunotherapy.

\section{Materials and methods}

Cell lines and cell cultures. The HEK 293 (human embryonic kidney), HT1080 (human fibrosarcoma), JC (mice breast cancer cells), HT2 (murine IL2/IL15 dependent) and YAC-1 (A murine T-lymphoma cell line sensitive to NK-cells) cells were purchased from Bioresource Collection and Research Center (BCRC, Shinchu, Taiwan) and cultured in DMEM or RPMI-1640 medium supplemented with $10 \%$ fetal bovine serum, and $100 \mathrm{IU} / \mathrm{ml}$ penicillin/streptomycin. In addition, HT2 cell line needs supplement with IL2 or equivalent IL15 protein (Santa Cruz Biotechnology, Santa Cruz, CA) for maintaining survival. DMEM, RPMI, fetal bovine serum, L-glutamine, penicillin/streptomycin, sodium pyruvate and non-essential amino acids were purchased from Invitrogen (Carlsbad, CA, USA).

Plasmids and plasmid construction. The rAAV2 helper-free system, containing packaging plasmid pAAV-MCS plasmid, pAAV-RC plasmid, pHelper, pAAV-hrGFP plasmid was purchased from Stratagene (La Jolla, CA). The plasmid containing human IL15 gene (hIL15) was provided though the kindness of Dr K.W. Liao (National Chiao Tung University, Taiwan). The hIL15 containing EcoR I and BamH I sites was amplified for cloning using the polymerase chain reaction. Oligonucleotide primers containing EcoRI and BamHI sites were synthesized as follows: sense primer: 5'-GAATTC AAA GAA TTC ATG TAC AGG ATG CAA CTC CT, and anti-sense primer, 3'-GGATCC AAA GGA TCC TTA AGA AGT GTT GAT GAA CAT TTG G. The PCR conditions used a thermal profile as described previously (21). The amplified hIL15 cDNA was inserted between the EcoRI and BamHI sites of pAAV-MCS to yield pAAV-hIL15 plasmid.
Production and purification of rAAV2. Production of rAAV2hIL15 and rAAV2-hrGFP was done in the helper-free system as described previously (22). For rAAV2-hIL15 production, 293 cells were cultured on fifty $15-\mathrm{cm}$ dishes and transfected with calcium chloride with $2 \mathrm{mg}$ pAAV-hIL 15 plasmid, $2 \mathrm{mg}$ pAAV-RC plasmid and $2 \mathrm{mg}$ pAAV-Helper plasmid. For rAAV2-hrGFP production, 293 cells were cultured on fifty $15-\mathrm{cm}$ dishes and transfected with calcium chloride with $2 \mathrm{mg}$ pAAV-hrGFP plasmid, $2 \mathrm{mg}$ pAAV-RC plasmid and $2 \mathrm{mg}$ pAAV-Helper plasmid. After $65 \mathrm{~h}$ of transfection, rAAV2hIL15 and rAAV2-hrGFP were produced in 293 cells. Purification of rAAV2-hIL15 and rAAV2-hrGFP was done using a single-step column purification (SSCP) method (23). The rAAV2 was concentrated to about $1 \mathrm{ml}$ with an Amicon Ultra-15 centrifugal filter (Millipore, Billerica, MA) and stored at $-80^{\circ} \mathrm{C}$, as described previously (21). Purified rAAV2 (rAAV2-hIL15 and rAAV2-hrGFP) was determined by loading $10^{10}$ viral particles on $10 \%$ SDS gel (23).

Measurement of viral titer and endotoxin activity of rAAV2. The viral titers of rAAV2 were determined by real-time PCR method as described previously $(21,24)$. Primers including forward primer (5'-GCC CAC TTG GCA GTA CAT CAA), reverse primer (3'-GGG CCA TTT ACC GTC ATT GAC) and probe primer (CAT ATG CCA AGT ACG CCC C) were designed by ABI Research (New York, NY). A total of $2-5 \times 10^{12}$ genomic particles of rAAV2 can be obtained from fifty $15-\mathrm{cm}$ dishes. The endotoxin of the produced rAAV2 vectors were determined using endotoxin kit (Cambrex, Walkersville, MD).

ELISA and in vitro bioactivity assay. To determine the resulting expression of protein and bioactivity of rAAV2-hIL15, the culture media from rAAV2-hIL15 (1013 viral particles/ml), empty-vector (1013 viral particles/ml) and transduced HT1080 cells $\left(1.5 \times 10^{5}\right.$ cells/well) placed on 6 -well plates for 3 days were collected and subjected to ELISA and bioactivity analysis. In the ELISA assay (Biosource, Camarillo, CA), hIL15 antibody was coated on 96-well plates at first then added to culture media. After $4 \mathrm{~h}$ of reaction and then measured at optical density of $450 \mathrm{~nm}$. In order to determine the bioactivity of rAAV2-hIL15, the culture medium from rAAV2-hIL15 or empty-vector transduced HT1080 cells were used to treat HT2 cells $\left(6 \times 10^{3}\right.$ cells/well) for $16 \mathrm{~h}$, and the cell viability score was measured using MTS assay (Promega, Madison, WI), used as the surrogate maker of rAAV2-hIL15 bioactivity. Media containing purified recombinant hIL15 at $1 \mathrm{~g} / \mathrm{ml}$ (Santa Cruz Biotechnology) were added to HT2 cells which served as positive control in the bio-activity assay.

Animal studies. The JC mouse breast cancer cells were used in animal experiments to monitor rAAV2-hIL15 antitumor activities. Female BALB/c mice (18-23 g/mouse, 4-6-weeks old) were obtained from the National Animal Laboratory Center (Taipei, Taiwan). All procedures were done in compliance with the standard operating procedures of the Laboratory Animal Center of Tzu Chi University (Hualien, Taiwan). Syngenic BALB/c mice (6 rats per group) were implanted subcutaneously on the back with $1 \times 10^{5} \mathrm{JC}$ cells. Six mice in the study group were treated with rAAV2 -hIL15 

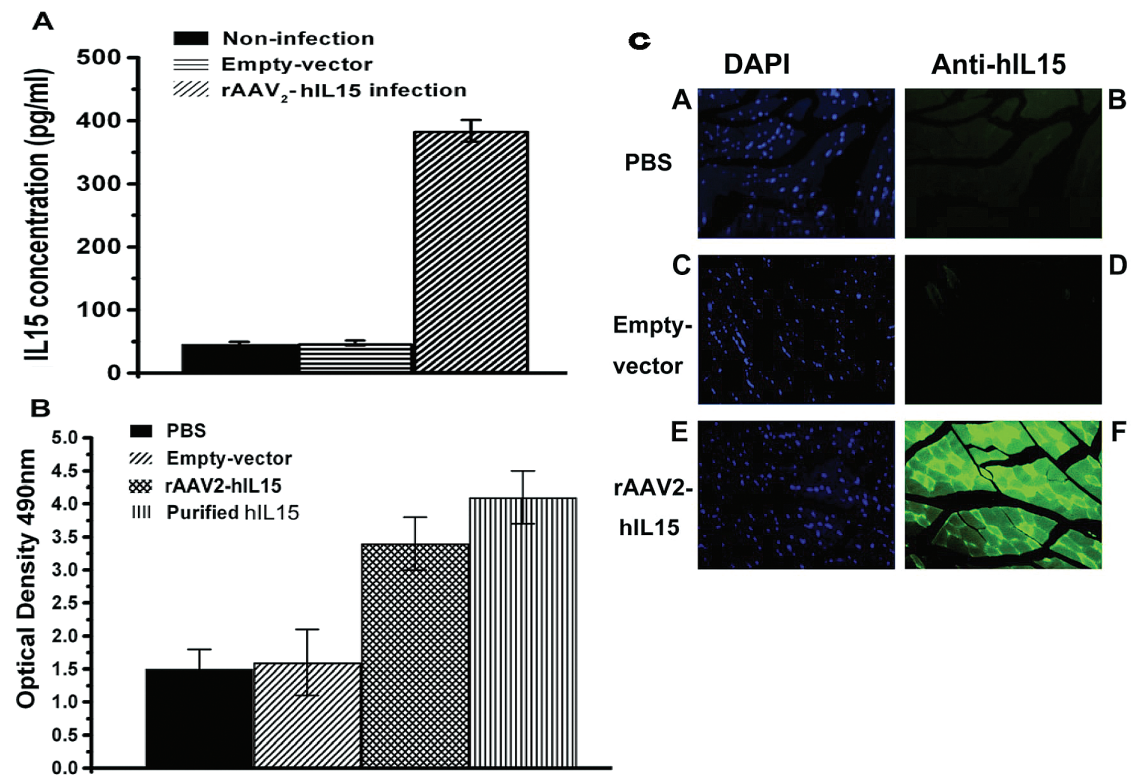

Figure 1. The expression and bioactivity of rAAV2-hIL15. (A) hIL15 protein production was measured by ELISA in rAAV2-hIL15, empty-vector and non-infected HT1080 cells. (B) Culture media obtained from the 3 groups above were added to HT2 cell culture for bioactivity assay. Purified hIL15 was added as positive control and cell viability is obtained by MTS assay. (C) Mice were sacrificed when tumor size exceeded $1500 \mathrm{~mm}^{3}$. The muscle of injection sites of all three groups were obtained for anti-hIL15 immunofluorescent stain (A, C and E, DAPI; B, D and F, anti-IL15 FITC).

(1013 viral particles (VP)/kg) by intramuscular injection over the quadricep muscle of the left thigh, four weeks prior to tumor cell implantation. The mice in the control group were treated with empty-vector $(1013 \mathrm{VP} / \mathrm{kg})$ or PBS at the same time comparatively. Body weights of all groups of mice were measured three times a week for basic safety examination. Tumor size was measured with the caliper three times a week and the tumor volume was calculated as $1 / 2 \mathrm{x}$ length $\mathrm{x}$ width ${ }^{2}$. Animals were sacrificed when tumor volume exceeded $1500 \mathrm{~mm}^{3}$ and tissue biopsy of the tumor, muscle and other vital organs were obtained, stained with H\&E and observed and photographed under a light microscope.

LAK cells cytotoxicity assay. rAAV2-hIL15 infects mice by intramuscular injection for $>9$ weeks. After the test animals were sacrificed and their blood collected, the lymphocytes were separated from dead cells and red blood cells by ACK lysis buffer $\left(0.15 \mathrm{mM} \mathrm{NH}_{4} \mathrm{Cl}, 1.0 \mathrm{mM} \mathrm{KHCO}_{3}, 0.1 \mathrm{mM}\right.$ $\mathrm{Na}_{2}$ EDTA, pH 7.3). Cytotoxic activity of LAK cells was determined by the lactate dehydrogenase (LDH) release assay using CytoTox 96 Non-Radioactive Cytotoxicity Assay kit (Promega). Target cells used in LAK cell cytotoxicity assay were YAC-1 cells which were incubated in a total volume of $200 \mu \mathrm{l}$ with effector cells in RPMI-1640 containing $10 \%$ FCS in 96-well round-bottom micro-titer plates at various cell densities in order to achieve effectorto-target $(\mathrm{E} / \mathrm{T})$ ratios $(1: 1,12.5: 1,25: 1$, and $50: 1)$ for $4 \mathrm{~h}$ at $37^{\circ} \mathrm{C}$. Target cell lysis was calculated as: (OD490 $\mathrm{nm}$ of sample - OD490 nm with spontaneous release of LDH from target cells - OD490 nm with spontaneous release of LDH from effector cells) x 100/(OD490 nm with maximum release of LDH from target cells - OD490 nm with spontaneous release of $\mathrm{LDH}$ from effector cells).

Immunofluorescent assay. The muscles of mice with rAAV2hIL15 (control) with injection sites over the left thighs were obtained for immunofluorescent staining. The muscles were fixed in $10 \%$ formalin, embedded in paraffin, then cut into $5-\mu \mathrm{m}$ sections and fixed in saline coating slides. Later, these slides were treated with $0.03 \%$ Triton-X-100 and blocked with $3 \%$ BSA. The slides were incubated with hIL15 antibody overnight at $40^{\circ} \mathrm{C}$ and then incubated with a secondary goatanti-rabbit-FITC antibody (Jackson ImmunoResearch, West Grove, PA) at room temperature for $1 \mathrm{~h}$. After mounted with cover slips, the slides with muscle slices were visualized and photographed under fluorescent microscopy.

In situ detection of apoptotic cells. Tumor specimens of all groups were obtained after each mouse was sacrificed when tumor volume exceeded $1500 \mathrm{~mm}^{3}$ to investigate whether rAAV2-hIL15 suppressed the tumor growth via programmed cell death or not. Apoptotic tumor cells were labeled by the TUNEL method (terminal deoxynucleotinyl transferasemediated dUTP nick-end labeling) using an in situ cell death detection kit from Roche Applied Science (Penzberg, Germany) following the manufacturer's recommendation.

Statistical analyses. Data were expressed as mean \pm SEM. Statistical significance was analyzed by Student's t-test. The survival analysis was done using the Kaplan-Meier method where $\mathrm{P}<0.05$ was considered to be statistically significant.

\section{Results}

The expression of rAAV-hIL15 protein in vitro and in vivo. We examine the hIL15 protein expression in vitro by using ELISA and HT2 viability assay. We measured the hIL15 protein titers by ELISA from transfected and non-transfected HT1080 cells (Fig. 1A). The results demonstrated that rAAV2-hIL15 transfected HT1080 cells expressed 8-fold increase in hIL15 proteins as compared with the non-transfected and empty-vector control groups. The hIL15 protein 
expression by rAAV2-hIL15 was further examined by HT2 cell culture system. HT2 cells are IL2 or IL15 dependent cells which require the IL2 or IL15 supplement to proliferate. Culture media obtained from the above 3 groups were added to HT2 cells in 96-well culture plates and incubated overnight for viability assay. The HT2 cell viability is observed with MTS assay and the purified hIL15 serves as the positive control. The group treated with media obtained from rAAV2-hIL15 infected HT1080 cells showed higher viability as compared with the control (Fig. 1B).

In order to investigate whether rAAV2-hIL15 could use muscle as a platform to produce hIL15 protein in vivo, the injection sites of quadriceps muscles of all groups of mice were obtained for immune-fluorescence assay after each mouse was sacrificed. The immune-fluorescence assay of muscle biopsy revealed hIL15 expression with green fluorescence over rAAV2-hIL15 injection sites under a fluorescent microscope; green fluorescence was not seen in negative control groups (Fig. 1C).

rAAV2-hIL15 delayed tumor appearance, suppressed tumor growth and prolonged survival of mice bearing subcutaneous JC breast cancer cells. We performed the in vivo animal experiments to investigate antitumor activity of rAAV2-hIL15 and there was a significant inhibitory effect on JC breast cancer cell growth for the rAAV2-hIL15 group compared with negative control groups (Fig. 2A). The onset of tumor in this study group was delayed for about 5 days as compared with negative control groups (time to develop a measurable tumor, rAAV2-hIL15 group at day 21 versus control group at day 16). The rAAV2-hIL15 suppressed tumor growth. The average tumor size of rAAV2-hIL15 group at day 35 was significantly smaller than control groups (Fig. 2A). To investigate whether rAAV2-hIL15 could prolong survival of tumor-bearing mice, all tumor-bearing mice were sacrificed when tumor volume exceeded $1500 \mathrm{~mm}^{3}$ and that day was used as the final survival day for that mouse. Survival of mice in the rAAV2-hIL15 group was longer then the control ones (Fig. 2B).

In order to investigate the safety of rAAV2-hIL15 single dose intramuscular injection, basic safety examination was performed by measuring body weights of all groups of mice as well as histological analysis. The heart, lung, liver, and kidney of all groups of mice were obtained after each mouse was sacrificed for histological analysis. Tissue sections were stained with hematoxylin-eosin, observed and photographed under a light microscope and thus described. We found no body weight or histological difference between the rAAV2hIL15 group and control group (data not shown).

Antitumor cytotoxic activity of LAK cells induced by rAAV2hIL15 in vivo. To test whether rAAV2-hIL15 could induce antitumor cytotoxic activity of LAK cells, we collected LAK cells derived from splenocytes of sacrificed mice against YAC-1 cells (target for LAK cells) with the lactate dehydrogenase (LDH) release assay using CytoTox 96 NonRadioactive Cytotoxicity Assay kit. The data suggested that antitumor cytotoxic activity of LAK cells can be induced in rAAV2-hIL15 group up to 4 -fold as compared with the control groups at effector/target ratio of 50:1 (Fig. 3).
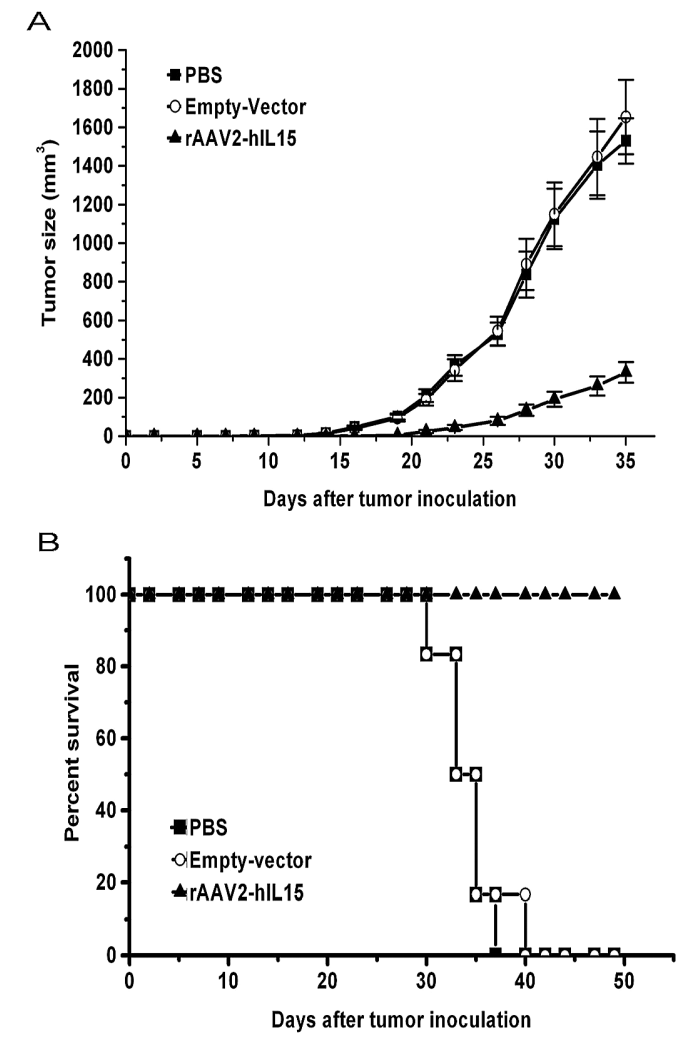

Figure 2. rAAV2-hIL15 delayed tumor appearance and suppressed tumor growth in vivo. (A) Tumor volumes were measured three times per week, calculated as $1 / 2 \times$ length $\mathrm{x}$ width $^{2}$, and represented as mean $\pm \mathrm{SEM}$. Each mouse was sacrificed when tumor volume exceeded $1500 \mathrm{~mm}^{3}$. (B) JC breast cancer cells $\left(1 \times 10^{5}\right)$ were inoculated subcutaneously into the right side of the flank region of BALB/c mice. Survival rate was monitored three times per week. Mice were sacrificed when tumor size exceeded $1500 \mathrm{~mm}^{3}$.

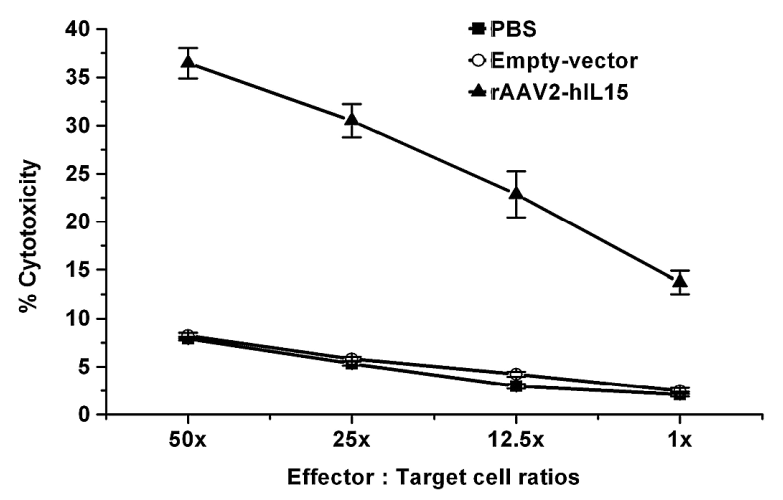

Figure 3. Cytotoxic activities of LAK cells were activated by rAAV2-hIL15 in vivo. The cytotoxtic activity of LAK cells which derived from splenocytes were assayed by using the lactate dehydrogenase $(\mathrm{LDH})$ release assay

rAAV2-hIL15 induction of apoptosis on tumor cells. To investigate whether rAAV2-hIL15 suppressed tumor growth via apoptosis or not, we obtained tumor specimens from all study groups to perform in situ labeling of fragmented DNA using the TUNEL method. Prominent apoptotic cells were detected in tumors treated with rAAV2-hIL15 as compared to negative control groups where no apoptosis was observed (Fig. 4). 


\section{TUNEL}
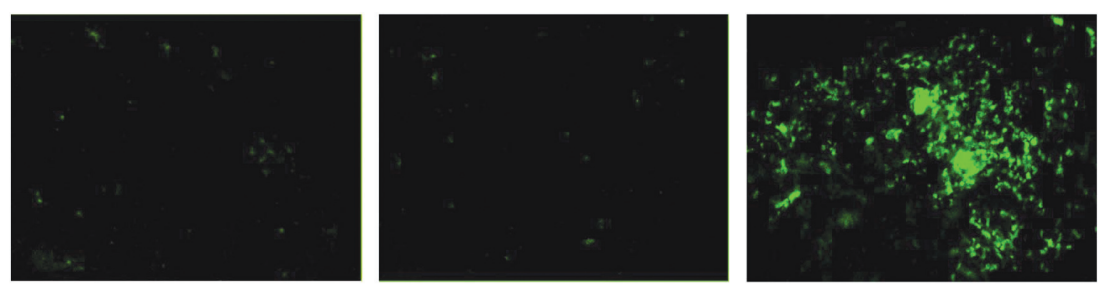

A PBS

\section{B Empty-vector C rAAV2-hIL15}

Figure 4. rAAV2-hIL15 pretreatment resulted in increased apoptosis of tumor cells. After the mice were sacrificed, the tumors were dissected in a horizontal plane, and sections were prepared and examined for apoptosis using TUNEL method (green fluorescence, x200) from tumors treated with PBS, empty-vector, and rAAV2-hIL15.

\section{Discussion}

As of 2007, over 1000 clinical trials in gene therapy have been initiated with $66.5 \%$ targeting cancer according to the worldwide website on gene therapy clinical trials. AAV vectors are more potent and safe than the previous two with lower immunogenicity than adenoviral vectors with no risk of randomized insertional gene mutation as compared with the retroviral vectors. In this study, we chose AAV vector carrying hIL15 gene to maintain long-term gene expression to stimulate LAK cells for breast cancer therapy. This method could avoid the obstacles of previous studies using cytokine proteins for tumor treatments because of their short lives and multiple doses are required (17).

AAV is a single-stranded DNA virus and must convert into a double stranded template for further gene transcription (25). This rate-limiting step takes about 3 weeks. In order to produce sufficient therapeutic proteins in mice, we designed the therapeutic protocol as rAAV2-hIL15 single dose (1013 $\mathrm{VP} / \mathrm{kg}$ ) intramuscular injection over the quadricep muscles for 4 weeks prior to tumor implantation.

Previous studies reported that hIL15 proteins share $\sim 73 \%$ sequence identity with murine IL15 and can be used by mice (26). In our study, we produced rAAV2 carrying hIL15 gene and successfully applied on a single dose intramuscular administration. Expression of hIL15 protein was observed through immunofluorescent assay over muscle injection sites.

The major drawback of using rAAV vector for gene therapy is difficult at large scale production of high bioactivity and high purity vectors. We built up a hospital-based current good manufacturing practice (cGMP) laboratory for gene vector production 5 years ago. We were able to successfully produce rAAV vectors via triple plasmid co-transfection by using AAV helper-free system. We modified the purification method by adding deoxycholic acid to remove extra DNA fragments and nucleotides, changed hitrap heparin column to heparin-agarose gel column according to single step column purification (SSCP) method so as to increase affinity to rAAV vectors. We were able to produce higher titers $(1013 \mathrm{VP} / \mathrm{ml})$ and higher purity rAAV vectors than previous purification method proved by real-time PCR and SDS-page (data not shown).

Of the 55\% adenoviruses that have neutralizing antibody (NAB) in human serum, only $32 \%$ AAVs have NAB (27). In a study done previously from the Institute of Human Gene Therapy of University of Pennsylvania, it was reported that high levels and stable transgene expression were found in mice following intramuscular injection of purified rAAV (28). The rAAV genome is efficiently incorporated into the nuclei of differentiated muscle fibers. Neutralizing antibody against AAV capsid proteins does not prevent readministration of the vector. No humoral or cellular immune responses are elicited in the neoantigenic transgene product (28). The favorable feature of rAAV in muscle-directed gene therapy showed great potential for this study to be applied in the treatment of cancer.

In this study, we were able to present evidence that a single dose intramuscular rAAV2-hIL15 injection is feasible. It mediates an effective antitumor immunity via activation of LAK cells, inducing tumor apoptosis and inhibiting JC breast cancer growth. These data provide a preclinical rationale for rAAV2-hIL15-based breast cancer immunotherapy.

\section{Acknowledgements}

This work was supported by grants from the Buddhist Tzu Chi General Hospital of Taiwan and by National Science Council of Taiwan grants NSC95-2320-B-241-007 to C.W.W., NSC96 2320-B-039-032-MY3 and NSC96 3111-B-039-003 to Y.L.Y, and NSC98 2320-B-197-002-MY3 to Y.L.C.

\section{References}

1. Ponnazhagan S, Curiel DT, Shaw DR, Alvarez RD and Siegal GP: Adeno-associated virus for cancer gene therapy. Cancer Res 61: 6313-6321, 2001.

2. Xiao X, Li J and Samulski RJ: Efficient long-term gene transfer into muscle tissue of immunocompetent mice by adeno-associated virus vector. J Virol 70: 8098-8108, 1996.

3. Ma HI, Guo P, Li J, et al: Suppression of intracranial human glioma growth after intramuscular administration of an adenoassociated viral vector expressing angiostatin. Cancer Res 62: 756-763, 2002.

4. Zhang JF, Hu C, Geng Y, Blatt LM and Taylor MW: Gene therapy with an adeno-associated virus carrying an interferon gene results in tumor growth suppression and regression. Cancer Gene Ther 3: 31-38, 1996.

5. Liu DW, Tsao YP, Kung JT, et al: Recombinant adeno-associated virus expressing human papillomavirus type 16 E7 peptide DNA fused with heat shock protein DNA as a potential vaccine for cervical cancer. J Virol 74: 2888-2894, 2000.

6. Hildinger M and Auricchio A: Advances in AAV-mediated gene transfer for the treatment of inherited disorders. Eur J Hum Genet 12: 263-271, 2004.

7. Emborg ME, Carbon M, Holden JE, et al: Subthalamic glutamic acid decarboxylase gene therapy: changes in motor function and cortical metabolism. J Cereb Blood Flow Metab 27: 501-509, 2007. 
8. Kaplitt MG, Feigin A, Tang C, et al: Safety and tolerability of gene therapy with an adeno-associated virus (AAV) borne GAD gene for Parkinson's disease: an open label, phase I trial. Lancet 369: 2097-2105, 2007.

9. Rao VS, Dyer CE, Jameel JK, Drew PJ and Greenman J: Potential prognostic and therapeutic roles for cytokines in breast cancer (Review). Oncol Rep 15: 179-185, 2006.

10. Dougan $M$ and Dranoff G: Immune therapy for cancer. Annu Rev Immunol 27: 83-117, 2009.

11. Carson WE, Giri JG, Lindemann MJ, et al: Interleukin (IL) 15 is a novel cytokine that activates human natural killer cells via components of the IL-2 receptor. J Exp Med 180: 1395-1403, 1994.

12. Munger W, DeJoy SQ, Jeyaseelan R Sr, et al: Studies evaluating the antitumor activity and toxicity of interleukin-15, a new T cell growth factor: comparison with interleukin-2. Cell Immunol 165: 289-293, 1995.

13. Mrozek E, Anderson P and Caligiuri MA: Role of interleukin-15 in the development of human $\mathrm{CD} 56^{+}$natural killer cells from $\mathrm{CD}^{2} 4^{+}$hematopoietic progenitor cells. Blood 87: 2632-2640, 1996.

14. Armitage RJ, Macduff BM, Eisenman J, Paxton R and Grabstein KH: IL-15 has stimulatory activity for the induction of B cell proliferation and differentiation. J Immunol 154: 483-490, 1995

15. Gamero AM, Ussery D, Reintgen DS, Puleo CA and Djeu JY: Interleukin 15 induction of lymphokine-activated killer cell function against autologous tumor cells in melanoma patient lymphocytes by a CD18-dependent, perforin-related mechanism. Cancer Res 55: 4988-4994, 1995.

16. Grimm EA, Robb RJ, Roth JA, et al: Lymphokine-activated killer cell phenomenon. III. Evidence that IL-2 is sufficient for direct activation of peripheral blood lymphocytes into lymphokine-activated killer cells. J Exp Med 158: 1356-1361, 1983.

17. Sparano JA, Fisher RI, Weiss GR, et al: Phase II trials of high-dose interleukin-2 and lymphokine-activated killer cells in advanced breast carcinoma and carcinoma of the lung, ovary, and pancreas and other tumors. J Immunother Emphasis Tumor Immunol 16: 216-223, 1994.
18. Lieberman J: The ABCs of granule-mediated cytotoxicity: new weapons in the arsenal. Nat Rev Immunol 3: 361-370, 2003.

19. Ferrini S, Azzarone B and Jasmin C: Is IL-15 a suitable candidate for cancer gene therapy? Gene Ther 3: 656-657, 1996.

20. Kimura K, Nishimura H, Hirose K, Matsuguchi T, Nimura Y and Yoshikai Y: Immunogene therapy of murine fibrosarcoma using IL-15 gene with high translation efficiency. Eur J Immunol 29: 1532-1542, 1999.

21. Yiang GT, Harn HJ, Yu YL, et al: Immunotherapy: rAAV2 expressing interleukin-15 inhibits HeLa cell tumor growth in mice. J Biomed Sci 16: 47, 2009.

22. Zolotukhin S, Byrne BJ, Mason E, et al: Recombinant adenoassociated virus purification using novel methods improves infectious titer and yield. Gene Ther 6: 973-985, 1999.

23. Auricchio A, Hildinger M, O'Connor E, Gao GP and Wilson JM: Isolation of highly infectious and pure adeno-associated virus type 2 vectors with a single-step gravity-flow column. Hum Gene Ther 12: 71-76, 2001.

24. Gao G, Qu G, Burnham MS, et al: Purification of recombinant adeno-associated virus vectors by column chromatography and its performance in vivo. Hum Gene Ther 11: 2079-2091, 2000.

25. Wang Z, Ma HI, Li J, Sun L, Zhang J and Xiao X: Rapid and highly efficient transduction by double-stranded adeno-associated virus vectors in vitro and in vivo. Gene Ther 10: 2105-2111, 2003.

26. Kutzler MA, Robinson TM, Chattergoon MA, et al: Coimmunization with an optimized IL-15 plasmid results in enhanced function and longevity of CD8 T cells that are partially independent of CD4 T cell help. J Immunol 175: 112-123, 2005.

27. Chirmule N, Propert K, Magosin S, Qian Y, Qian R and Wilson J: Immune responses to adenovirus and adeno-associated virus in humans. Gene Ther 6: 1574-1583, 1999.

28. Fisher KJ, Jooss K, Alston J, et al: Recombinant adenoassociated virus for muscle directed gene therapy. Nat Med 3: 306-312, 1997. 